\title{
Breast Lesions of Uncertain Malignant Potential: A Challenge for Surgeons
}

\author{
Deslauriers Nancy ${ }^{1}$, Sidéris Lucas ${ }^{1}$, Dufresne Michel-Pierre ${ }^{2}$, Mitchell Andrew ${ }^{3}$, Drolet Pierre ${ }^{4}$, Dubé Pierre ${ }^{1}$, \\ Leclerc Yves E. ${ }^{1} \&$ Leblanc Guy ${ }^{1}$ \\ ${ }^{1}$ Department of surgical oncology, Hospital Maisonneuve-Rosemont- University of Montreal, Quebec, Canada \\ ${ }^{2}$ Departement of radiology, Hospital Maisonneuve-Rosemont- University of Montreal, Quebec, Canada \\ ${ }^{3}$ Department of pathology, Hospital Maisonneuve-Rosemont- University of Montreal, Quebec, Canada \\ ${ }^{4}$ Department of anesthesiology, Hospital Maisonneuve-Rosemont- University of Montreal, Quebec, Canada \\ Correspondence: Nancy Deslauriers, M.D. Hospital Maisonneuve-Rosemont- University of Montreal, 5415 \\ boulevard de l'Assomption, Montreal, Quebec, Canada. Tel: 1-514-252-3400 \#4558. Fax:1-514-252-1463. Email: \\ Nancy.deslauriers@umontreal.ca
}

Received: March 28, 2012 Accepted: April 13, 2012 Online Published: May 1, 2012

doi:10.5539/cco.v1n1p77 URL: http://dx.doi.org/10.5539/cco.v1n1p77

\section{Mini Abstract}

Lobular neoplasia, radial scar and atypical ductal hyperplasia on core needle biopsy pose a challenge for surgeons. We looked at upgrade rates for these lesions to determine which should be excised. We found that lobular carcinoma in situ and atypical ductal hyperplasia had to be operated and that radial scar and atypical lobular hyperplasia could be observed.

\section{Structured Abstract}

Objective and background: The need for surgical resection of breast lesions of uncertain malignant potential diagnosed by core-needle biopsy (CNB) is not well established. Our study sought to determine the incidence of these lesions at CNB and to find the rate of pathologic upgrade at surgery. and during clinical follow-up. Method: A retrospective review of CNBs performed at our hospital covering the period from November 1999 to December 2008 was undertaken. In those patients diagnosed with lobular neoplasia (atypical lobular neoplasia (ALH) and lobular carcinoma in situ (LCIS)), atypical ductal hyperplasia (ADH) and radial scar (RS) the rates of pathologic upgrade at subsequent surgery were determined. Results: 9325 breast CNBs were performed during the period under review. There were 36 diagnoses of ALH (0.4\%), 20 of LCIS $(0.2 \%), 110$ of ADH (1.2\%) and 60 of RS (0.6\%). Regarding lobular neoplasia, ALH surgical resection was performed with subsequent pathologic upgrade in only $1 / 31$ patients $(3.2 \%) .85 \%$ of LCIS diagnoses were followed by surgical resection with pathologic upgrade in 5/17 cases (29\%). For patients with ADH, 51\% (56/110) had surgical resection with an upgrade rate of $20 \%(11 / 56)$. 9/54 patients (17\%) who did not undergo surgical resection subsequently developed a cancer at the biopsy site. Regarding RS, in 58\% (35/60) of cases surgical resection was undertaken with a pathologic upgrade rate of $8 \%(3 / 35)$. In all three cases with an upgrade the biopsy had been performed on a hypoechogenic lesion. Conclusion: We conclude that the significant rate of pathologic upgrade in LCIS and $\mathrm{ADH}$ mandates a surgical excision when these lesions are found at CNB. The low rate of pathologic upgrade in $\mathrm{RS}$ in the absence of a hypoechogenic lesion and ALH may allow these cases to be observed.

Keywords: breast, atypical ductal hyperplasia, atypical lobular hyperplasia, lobular neoplasia, upgrade, radial scar

\section{Introduction}

Breast cancer is one of the most prevalent cancers in women in the industrialised world. With the implantation of screening programs core-needle biopsy (CNB) has become the primary method of sampling microcalcifications discovered by mammography (Pagni et al., 2012). As more premalignant breast lesions are diagnosed by CNB, management challenges for surgeons are increasing. Hunt et al. reported a mean malignancy rate of $29 \%$ for Birads 3 lesions (Hunt, Steel, Porter, Holgate, \& Watkins, 2012). These premalignant lesions include lobular neoplasia, (atypical lobular hyperplasia (ALH) and lobular carcinoma in situ (LCIS)), atypical ductal hyperplasia $(\mathrm{ADH})$ and radial scar (RS). When these lesions are subsequently surgically resected, there is discovery of a 
higher grade lesion, including invasive carcinoma, ("pathological upgrade") in a varying number of specimens. As pathologic upgrade occurs in a minority of cases, there is a desire to avoid overtreatment of these lesions. However, a lack of consensus regarding management of these premalignant breast lesions persists to this day.

Lobular intraepithelial neoplasia (LIN) includes the entities of atypical lobular hyperplasia (ALH) and lobular carcinoma in situ (LCIS) (Haagensen, Lane, Lattes, \& Bodian, 1978). ALH and LCIS represent a spectrum of lesions characterized by proliferation of small and loosely cohesive cells originating in the terminal duct lobular unit. LIN is almost always found incidentally, is non palpable, mammographically nonspecific, frequently bilateral, and may develop into invasive cancer. As many as one third of women with LIN will develop invasive lobular or ductal cancer in either breast (Tavassoli FA, 2009).

$\mathrm{ADH}$ is an epithelial proliferation similar to low-grade ductal carcinoma in situ (DCIS) but without the uniform cytologic atypia and minimum size criteria which permit diagnosis of the latter. These histologic similarities suggest that ADH and DCIS represent a continuum of intraductal proliferation (Purcell \& Norris, 1998). Also, $\mathrm{ADH}$ is associated with a high incidence of pathologic upgrade when submitted to complete excision, with a mean rate of $44 \%$ (range: 11 to 58\%) (Jackman, Birdwell, \& Ikeda, 2002). The presence of ADH is associated with a risk factor of four to five of developing breast cancer (Schnitt SJ, 2009). The relative risk of later invasive breast cancer with atypical hyperplasia has been found to be 5 in the Nashville breast cohort (Sanders et al., 2006).

RS is a breast lesion that can mimic carcinoma, both clinically and radiographically The mammogram usually shows a "black star" consisting of a radiolucent center area with radiating long spicules. Histologically a fibroelastotic core with entrapped ducts is surrounded by radiating proliferating ductal elements (Rosen, 2001). Its significance in breast biopsies is still controversial. The coexistence of RS in specimen containing breast cancer has led to the hypothesis that RS may be an early phase of a malignant lesion. One study concluded that RS was an independent risk factor of cancer (Jacobs, Byrne, Colditz, Connolly, \& Schnitt, 1999). In this study, the authors compared RS associated with proliferative disease without atypia (PDWA) compared with PDWA alone and found an additional risk of carcinoma attributed to RS. In contrast, Berg and al., analysing the Mayo Benign Breast Disease data in a large retrospective study, found that the presence of RS did not increase the risk of breast cancer compared to PDWA alone (Berg et al., 2008). Such conflicting studies have created a challenge for surgeons in the management of RS.

The aims of our study were: 1) to determine the percentage of lesions diagnosed by CNB as ALH, LCIS, RS and $\mathrm{ADH}, 2)$ to determine the incidence of pathologic upgrade in those lesions surgically resected, and 3) to determine the re-biopsy rate and the number of late upgrades of those lesions that were not initially operated. Based on these results, we have proposed a management algorithm for these lesions.

\section{Material and Methods}

We retrieved all breast CNBs performed at Maisonneuve-Rosemont hospital from November 1999 to December 2008, with pathologic diagnoses of ALH, LCIS, ADH and RS. Retrospective reviews of clinical, radiology, pathology and surgical records were performed using data from patient files and the hospital clinical database system. CNB had been performed by stereotatic or ultrasound guidance. Stereotactic-guided core biopsy was performed with an 11-gauge directional vacuum-assisted biopsy device (Mammotome Biopsys, Johnson and Johnson Ethicon Endo-Surgery, Cincinnati, $\mathrm{OH}$ ). Ultrasound-guided core biopsy was performed with a spring-loaded biopsy device, using a 14 and 16 gauge Tru-cut needle. In cases of mammographically detected calcifications, a specimen radiograph confirmed the presence of calcifications in each sample, and a clip was left to mark the biopsy site.

All breast core needle biopsies had been analyzed by our surgical pathologists using established diagnostic criteria (Tavassoli, 2009; Schnitt, 2009). Patients were excluded if they had a previous history of breast cancer in the same breast quadrant or if the biopsy showed simultaneous proliférative malignant lesion in the same specimen.

Routine clinical practice in our center had been to recommend surgery for patient with LCIS, ADH and RS on core needle biopsy. As for ALH, other risk factors were considered such as the presence of residual calcification, family history and clinical examination. Patients who underwent surgical excision had a guidewire placed under stereotactic guidance in the site of the previous biopsy the morning of the surgery. After excision, specimen radiography was done to ensure that the marker, the guidewire and calcifications were all included. Standard gross and microscopic pathological examination followed. "Pathologic upgrade" was defined as the presence of any of DCIS, invasive ductal carcinoma or invasive lobular carcinoma in the resected surgical specimen. When found, the initial CNB was reviewed to confirm the initial premalignant diagnosis. Those patients who were not 
operated were followed with annual mammography and physical examination .When microcalcifications progressed or a new suspicious lesion was detected, further CNB was performed.

\section{Results}

From November 1999 to December 2008, 9325 breast CNBs were performed in our institution. A total of 252 cases of LIN, ADH and RS were diagnosed. Biopsies showing concomitant malignant neoplasia were excluded (26). Therefore, a total of 226 were retained for further analysis. The total prevalance of thoses diseases was $2,4 \%$. All occurred in women aged from 37 to 89 years with a mean of 56 years (SD:9). All patients were followed for a mean of 27 months (range:0-105, SD:24). See figure 1.

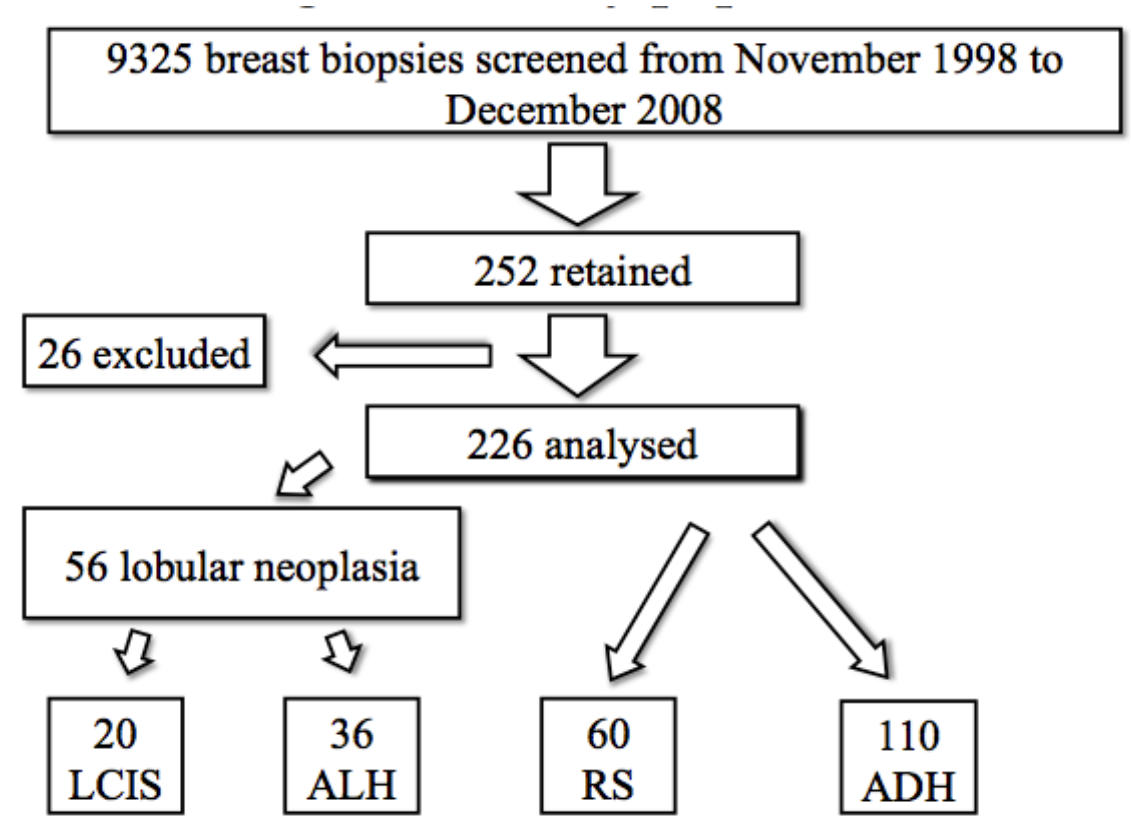

Figure 1. Study population

LCIS =lobular carcinoma in situ, ALH= atypical lobular hyperplasia; $\mathrm{RS}=$ radial scar; $\mathrm{ADH}=$ atypical ductal hyperplasia

\subsection{Atypical Lobular Hyperplasia}

36 cases of ALH were analysed (0.4\%). The mean age was 54 years (range:42-85, SD:8) and the mean follow-up was 31 months (range: 1-88, SD:24 months). Surgery was performed in ten patients (28\%). Only one patient $(10 \%)$ had a pathologic upgrade (invasive lobular carcinoma with 2 positive axillary lymph nodes); the lesion had not been detected initially on the mammogram but in retrospect was visible on ultrasound. She had initially presented with a suspicious palpable mass but a normal mammogram. Out of the 26 remaining patients, six required an additional $\mathrm{CNB}$ at the same site during follow-up. One patient underwent surgical excision for progression of microcalcifications and with subsequent discovery of LCIS. Only one patient developed invasive ductal carcinoma seven years after the original biopsy at the same site. Finally, another patient developed invasive ductal carcinoma in the contralateral breast one year after the biopsy. See figure 1A. 


\begin{tabular}{|c|c|c|c|c|}
\hline $\begin{array}{c}\text { ALH } \\
\text { biopsy }\end{array}$ & $\begin{array}{c}\text { Surgical } \\
\text { excision }\end{array}$ & Initial upgrade & Neoplasia & Follow up \\
\hline 36 & 10 yes & $1(10 \%)$ & 1 invasive & \\
\cline { 2 - 5 } $\begin{array}{c}0,4 \% \\
\text { incidence }\end{array}$ & 26 no & & $\begin{array}{c}1 \text { invasive } \\
7 \text { years after }\end{array}$ \\
\hline
\end{tabular}

\section{ALH}

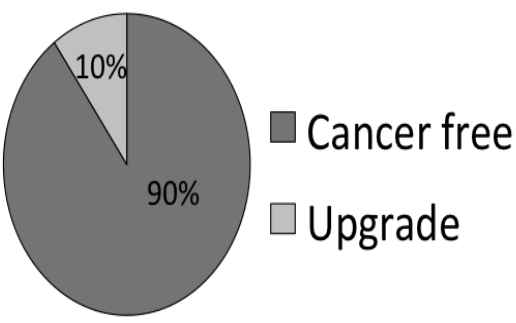

Figure 1A. ALH results

\subsection{Lobular Carcinoma In Situ}

20 classic LCIS were analysed $(0.2 \%$ of the population) None were of the pleomorphic variant. The mean age was 56 years (range:48-78, SD:9). 17 patients (85\%) had surgical excision and were followed for a mean of 24 months (range:3-96, SD:24). Two patients were lost to follow-up following biopsy and one refused surgery The mean delay between biopsy and surgery was three months. Five patients $(29 \%)$ had a pathologic upgrade following initial surgery. Of these, only one had a palpable mass and four had residual microcalcifications (80\%) following biopsy. Two patients had DCIS, (of which one developed invasive ductal carcinoma in the contralateral breast two years later), one had invasive lobular carcinoma and two had invasive ductal carcinoma. One patient with bilateral LCIS on initial biopsy underwent bilateral partial mastectomy. The right breast showed no additional pathology but in the left minimally invasive $(0.1 \mathrm{~cm})$ ductal carcinoma was found. Five years later, she developed advanced invasive ductal carcinoma in the right breast despite close follow up. All patients without any upgrade were followed and none developed further carcinoma. In all patients who underwent biopsy and surgery, 11 had residual calcifications: $7 / 13$ were without upgrade $(53 \%)$ and $4 / 5$ patients had a pathologic upgrade (80\%). See figure 1B.

\begin{tabular}{|c|c|c|c|c|}
\hline $\begin{array}{c}\text { LCIS } \\
\text { biopsy }\end{array}$ & $\begin{array}{c}\text { Surgical } \\
\text { excision }\end{array}$ & Initial upgrade & Neoplasia & Follow up \\
\hline 20 & 17 yes & $5(29 \%)$ & $\begin{array}{c}3 \text { invasivel } \\
2 \text { in situ }\end{array}$ & $\begin{array}{c}2 \text { controlateral } \\
\text { breast cancers }\end{array}$ \\
\cline { 2 - 5 } $\begin{array}{c}(0,2 \% \\
\text { incidence })\end{array}$ & $\begin{array}{c}3 \text { lost to } \\
\text { follow up }\end{array}$ & $\begin{array}{c}12 \text { without } \\
\text { cancer }\end{array}$ & & \\
\hline
\end{tabular}

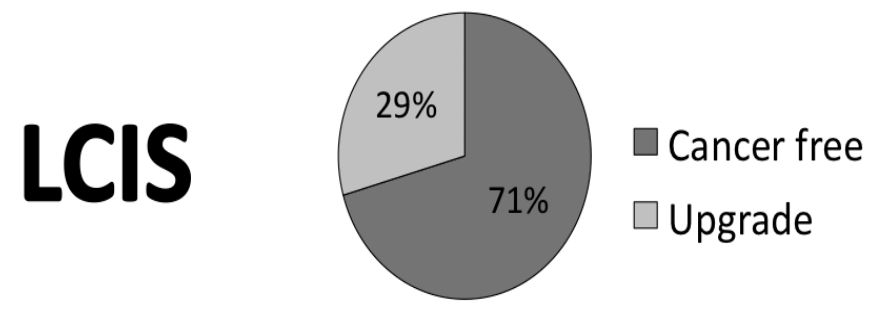

Figure 1B. LCIS results 


\subsection{Atypical Ductal Hyperplasia}

The ADH cohort consisted of 110 patients (1.2\%). All patients were followed for a mean of 31 months (range:1-105, SD:30). 17 patients were lost to follow up or decided to have their surgery in another hospital. After the first CNB, $56(51 \%)$ underwent surgery. There were 11 initial pathologic upgrades $(20 \%)$ Seven patients had DCIS and four patients had invasive ductal carcinoma. Of these upgraded patients, 8 had residual microcalcifications.and two had palpable lesions, which both revealed invasive ductal carcinoma. The mean age of our early upgrades was 55,5 and 57,5 for the others. The remaining 37 were clinically followed. 25 patients (68\%) required a second biopsy at the same site. 9 patients (18\%) were found to have carcinoma at the site of original biopsy after a mean time of 27 months (range:13-105, SD:30). 8 patients developed DCIS and one patient developed invasive ductal carcinoma. All underwent partial mastectomy. Of these 9 patients, 8 (89\%) had residual microcalcifications in the first $\mathrm{CNB}$ that contained $\mathrm{ADH}$. Three more patients required surgery for progression of microcalcifications which revealed benign pathology. A total of 68 patients $(62 \%)$ were operated overall at the site of the biopsy that contained ADH. A total of 20 patients $(22 \%)$ were found to have carcinoma after a biopsy diagnosis of ADH in a mean time of 27 months (Range:3-105, SD:24). See figure 1C.

\begin{tabular}{|c|c|c|c|c|}
\hline $\begin{array}{c}\text { ADH } \\
\text { biopsy }\end{array}$ & $\begin{array}{c}\text { Surgical } \\
\text { excision }\end{array}$ & Upgrade & Neoplasia & Follow up \\
\hline $\mathbf{1 1 0}$ & $\begin{array}{c}\mathbf{5 6} \text { yes } \\
\mathbf{( 5 1 \% )}\end{array}$ & $\begin{array}{c}\mathbf{1 1} \text { initial } \\
\mathbf{( 2 0 \% )}\end{array}$ & $\begin{array}{c}4 \text { invasive } / \\
7 \text { in situ }\end{array}$ & \\
\cline { 2 - 6 } & $\begin{array}{c}37 \text { no } \\
17 \text { lost }\end{array}$ & $\begin{array}{c}\mathbf{9} \text { late } \\
\mathbf{( 1 1 \% )}\end{array}$ & $\begin{array}{c}1 \text { invasive } / \\
8 \text { in situ }\end{array}$ & $\begin{array}{c}\text { 25 re-biopsies (68\%) } \\
\text { 12 late } \\
\text { surgeries (32\%) }\end{array}$ \\
\hline
\end{tabular}

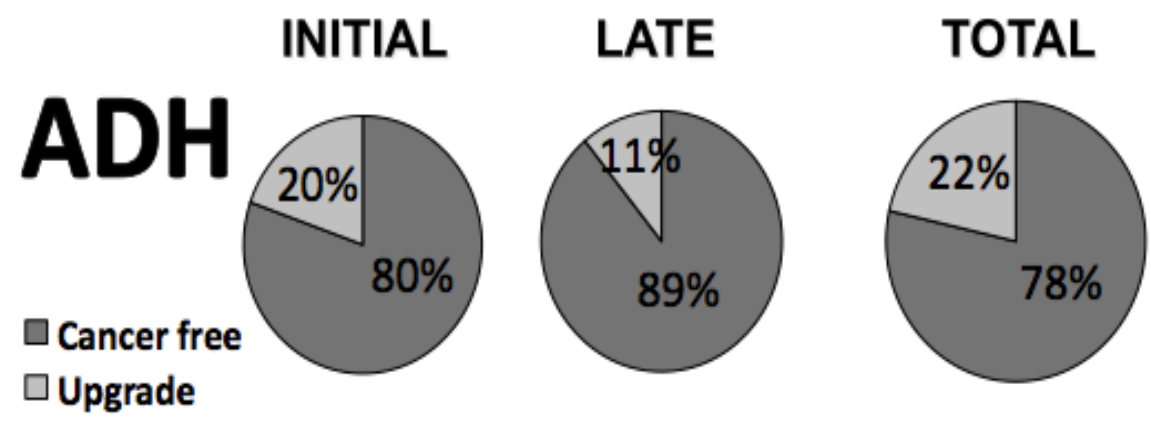

Figure 1C. Results of ADH

\subsection{Radial Scar}

60 cases of radial scar without atypia were diagnosed by CNB $(0.6 \%)$. The mean followup was 25 months (range:1-72, SD:20). A total of 35 patients underwent surgical excision(58\%) of which three had an initial pathologic upgrade. Two had DCIS and one had invasive ductal carcinoma. All three had presented with a hypoechogenic lesion of at least $9 \mathrm{~mm}$, and the biopsy was done under ultrasonographic guidance. None had any palpable mass and all had residual microcalcifications. Six patients needed a second biopsy at the original radial scar site because of progression of microcalcifications and one patient required a third. Of the 60 cases of RS, five were hypoechogenic lesions including all three in which breast carcinoma developed. See figure 1D. See figure 2 for a summary of the results. 


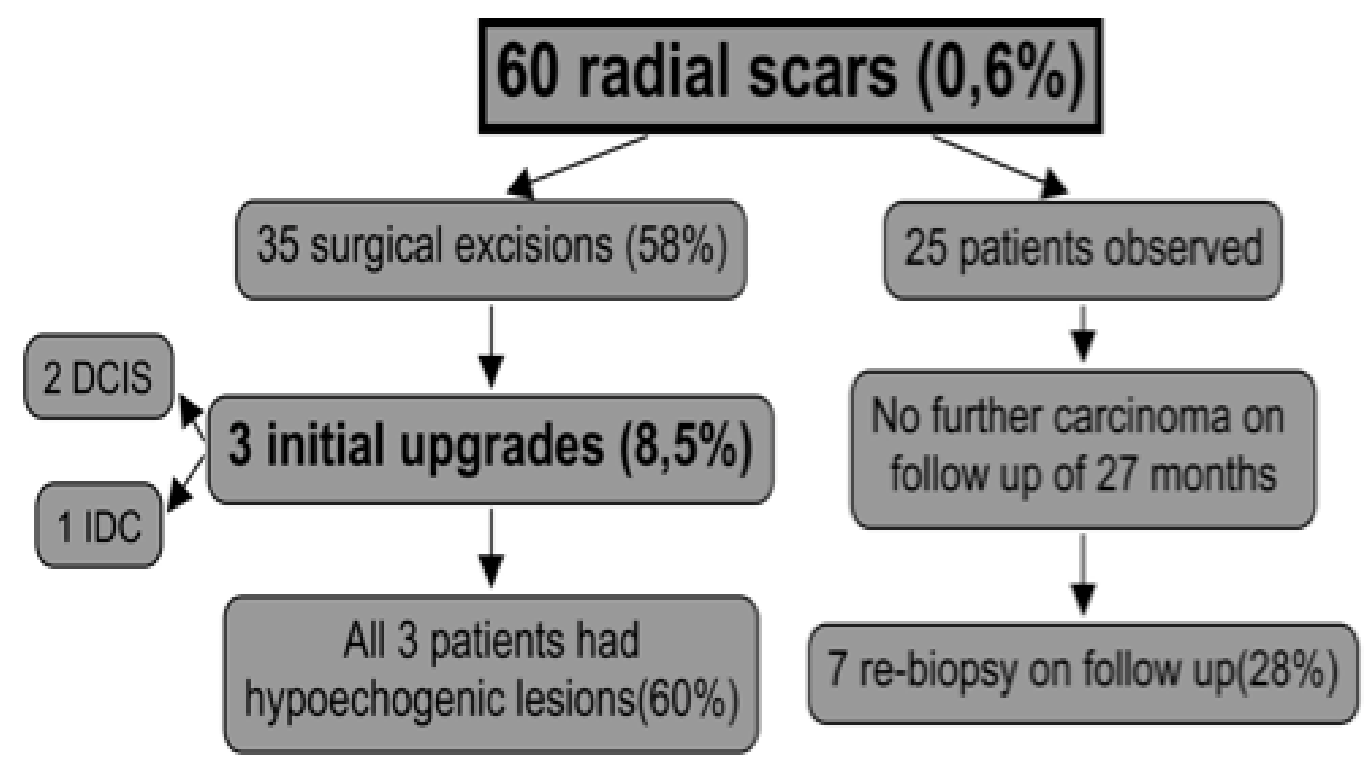

Figure 1D. Results of RS

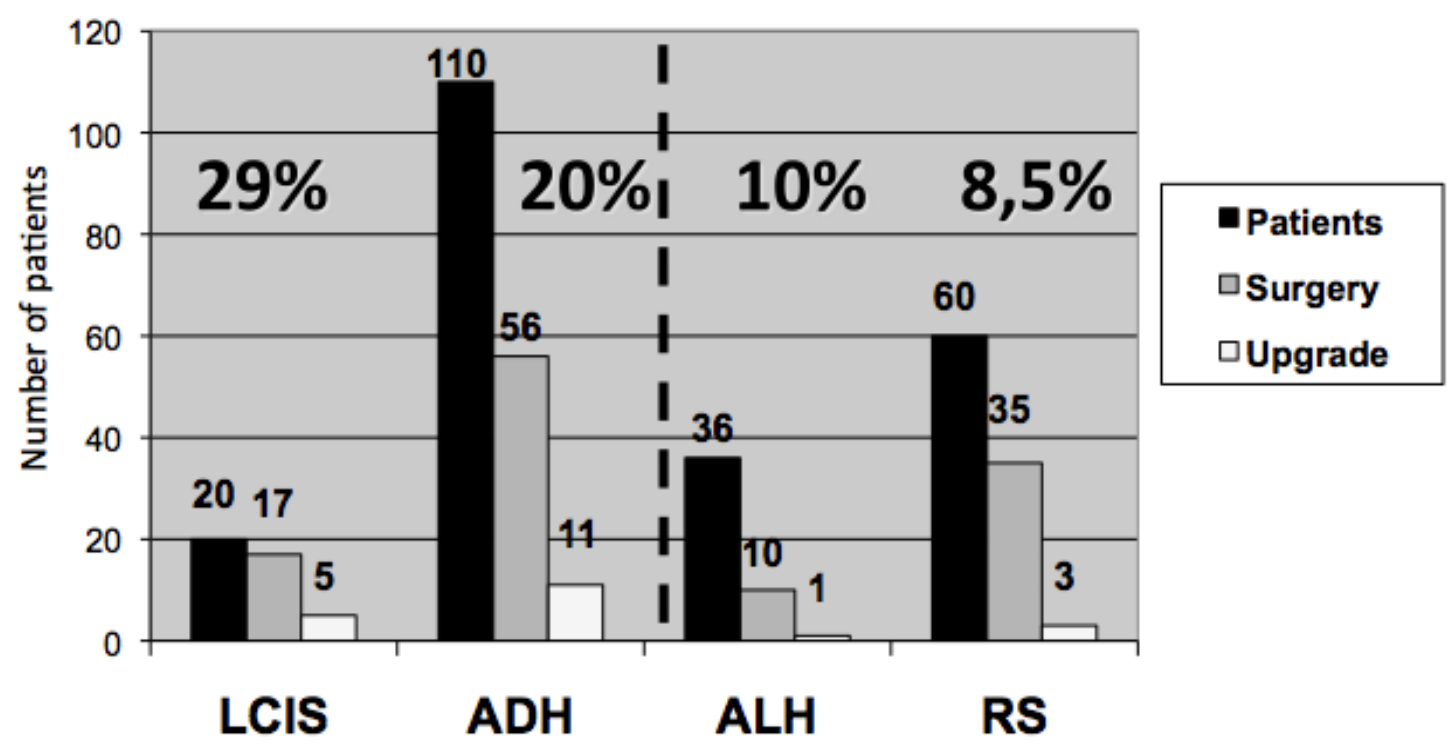

Figure 2. Number of patients, surgeries and initial upgrades in LCIS, ADH, ALH and RS

Description of number of patients, number of surgical excision and initial upgrade in lobular neoplasia, radial scar and atypical ductal hyperplasia. We note here a doted line which separates patients in need of surgical excision (left) vs expectative management(right) in regards to initial surgical upgrades. LCIS =lobular carcinoma in situ, $\mathrm{ALH}=$ atypical lobular hyperplasia $; \mathrm{RS}=$ radial scar ; $\mathrm{ADH}=$ atypical ductal hyperplasia 


\section{Discussion}

\subsection{Lobular Neoplasia}

Lobular neoplasia includes the spectrum of ALH and LCIS (Haagensen, et al., 1978). The histologic criteria proposed to distinguish these two entities have been found to have no prognostic significance (Tavassoli, 2009). Many surgeons believe that both pathologies may be managed similarly (Elsheikh \& Silverman, 2005). However, Page and al. demonstrated that the risk of developing IBC after a diagnosis of ALH is 4 to 5 times increased compared to 11 times increased in LCIS (Page, Kidd, Dupont, Simpson, \& Rogers, 1991). As there are no clear management guidelines for these diagnoses when found at $\mathrm{CNB}$, we analysed both pathologies separately. We found a pathologic upgrade rate of $10 \%$ in our ALH population. This finding is consistent with the literature as the rate of finding IBC at surgical excision varies from 0 to $67 \%$ (mean of 13\%) (Cangiarella et al., 2008). Our only patient with an upgrade (invasive ductal carcinoma) had a palpable mass with echographic traduction of the lesion. This is in keeping with the study of Middleton and al in which all 4 of ALH upgrades $(4 / 17 ; 24 \%)$ presented with a mass on physical exam.(Middleton, Perkins, Tucker, Sahin, \& Singletary, 2007) In the light of this low rate of pathologic upgrade, we propose that ALH in the absence of a mass and with negative echographic findings may be clinically followed.

Our higher rate of upgrade in the LCIS population, 29\%, leads us to conclude it is a more aggressive lesion. Similarities exist between lobular invasive carcinoma and adjacent lobular carcinoma in situ: the same mutation in the E-cadherin gene has been found in both, suggesting it may play an early role in tumorogenesis (Vos et al., 1997). Chuba and al. found a risk of developing invasive breast cancer with LCIS of $7.1 \%$ at 10 years (Chuba et al., 2005). As for surgical excision, the studies are contradictory (Brogi, Murray, \& Corben, 2010). Liberman and al. found an upgrade rate of $21 \%$ overall in 14 cases of LCIS and all upgrades were associated with high risk lesions (radial scar or atypical ductal hyperplasia) or histologic features that overlapped DCIS versus $0 \%$ upgrade in 5 LCIS cases without other findings (Liberman et al., 1999). They established that surgical excision was only needed when the biopsy showed histologic features overlapping those of DCIS, when a high-risk lesion was present or when a mammographic-histologic discordance was present. However, the small number of cases mandated a larger study. Elsheikh and al. studied 13 cases of LCIS and found 4 upgrades (31\%). They recommended surgical excision because of the significant rate of underestimation at biopsy.(Elsheikh \& Silverman, 2005) However, 2 pleomorphic LCIS were included in this study which may have overestimated the upgrade rate. Lavoué and al. showed, in their series, an upgrade rate between classic (7/42) and pleomorphic $(5 / 10)$ LCIS that was statistically different $(\mathrm{p}=0.003)$ (Lavoue et al., 2007). Of note, in our study, none of the patients had pleomorphic LCIS.

In light of our review of 20 cases of LCIS showing an upgrade rate of $29 \%$ and our review of the litterature, we recommend surgical excision when these lesions are diagnosed by CNB. Londero and al. calculated a positive predictive value of $57 \%$ for malignancy with LCIS (12/21) and of 7\% with ALH (1/14) with a statistically significant difference (Londero et al., 2008). They suggest that when LCIS is found at biopsy, a lesion larger than $20 \mathrm{~mm}$ or visible on ultrasound had a higher risk of harbouring cancer. In our study, only one patient presented with a mass that was visible on ultrasound. All others were random findings due to microcalcifications seen on mammography. Four of our upgrades $(80 \%)$ were associated with residual microcalcifications compared to only $53 \%$ for those without. This higher rate leads us to believe that residual microcalfications after biopsy may be a risk factor for further development of breast cancer.

\subsection{Radial Scars}

Radial scars are a pathological finding of unknown malignant potential. Previous studies have had contradictory conclusions. Jacobs and al. in the Nurse Health Study found that RS was an independent risk factor for breast cancer (Jacobs, et al., 1999). He determined that there was a relative risk (RR) of 1.8, regardless of associated histologic features. The presence of radial scar and proliferative disease without atypia (PDWA) had a relative risk of 3 compared to 1.5 with PDWA alone. He concluded that RS is an independent risk factor for breast cancer. Sanders and al. later used the Nashville Breast Cohort followed for a mean time of 20,4 years to study RS. He found a similar relative risk for breast cancer of 1,82 (Sanders, et al., 2006). However, in that study RS was usually associated with proliferative changes that could largely be attributed to the increased risk of carcinoma. He concluded that RS alone minimally increases the risk of IBC. Berg and al. analysed the Mayo Benign Breast Cohort and found a RR of 1,88 for RS with PDWA for IBC (Berg, et al., 2008). There was no statistical difference when compared to PDWA alone. He concluded that RS does not increase the risk of breast cancer.

However, it is noteworthy that these three studies included patients diagnosed before widespread mammographic screening was the norm, which may have underestimated the number of DCIS presenting with histologic RS. 
Our population was quite recent and all included patients were in the years of our province established breast screening program. Such screening methods may have increased the detection of DCIS presenting in the form of microcalcifications in our biopsies. Because patients with such findings at original biopsy were excluded, this may explain why we did not find late upgrades. Also, these studies did not mention the time interval between the diagnosis of RS and the presence of breast cancer.

Banchi and al. reported a rate of immediate upgrade of $8 \%$ for radial scars (Bianchi et al., 2012). In our study, we found similarly that only $8 \%$ of women with radial scar on biopsy had an upgrade to IBC at initial surgical excision. This is also consistent with the findings of Sanders who found an overall incidence of IBC associated with RS of 7\% (Sanders et al., 2006). The three patients all had a hypoechogenic lesion on ultrasound, whereas of all 60 women studied, only two others had such findings. Thus, sixty percent $(60 \%)$ of patients with a hypoechogenic lesion developed IBC. In the absence of a hypoechogenic lesion no cases of IBC developed. Egyed and al have shown a statistically significant difference between the "malignant" and "benign" ultrasound appearances of RS (Egyed et al., 2008). These findings lead us to suggest that women with histological diagnosed RS without atypia should undergo breast ultrasound. If the RS is visible on the ultrasound it should be surgically resected. Otherwise, these lesions may be clinically followed.

\subsection{Atypical Ductal Hyperplasia}

In our study, we found that $17 \%$ of our patients with $\mathrm{ADH}$ on $\mathrm{CNB}$ developed IBC in a mean time of 30 months (Range:1-105, SD:30), and an early upgrade rate of $18 \%$ Those results are consistent with reported rates which vary from 11 to $58 \%$ with a mean of $44 \%$ (Jackman et al., 2002). The most common uncertain finding mandating surgery at core needle biopsy was ADH in an Autralian breast screening program and the upgrade rate was $48 \%$ (Flegg, Flaherty, Bicknell, \& Jain, 2010). Liberman and al proved that $38 \%$ of ADH in vacuum-assisted breast biopsies had a pathologic upgrade at surgical excision (Liberman et al., 2007). This higher rate could be partly explained by the fact they reviewed biopsies prompted by abnormal MRI findings, which may select patients with higher risk factors. Jackman and al. studied ADH diagnosed using 11-gauge vacuum-assisted biopsies to determine if some factors could predict a low risk of carcinoma at surgical excision (Jackman et al., 2002). Their incidence of carcinoma was similar to ours, with $22 \%$ surgical resection specimens for ADH showing IBC. The authors noted three factors associated with a lower risk of upgrade: no personal history of breast cancer, lesions smaller than $1 \mathrm{~cm}$, and no residual lesion after biopsy. Our findings were similar: $80 \%$ of our early upgrades and $89 \%$ of our late upgrades had residual microcalcifications. An interesting finding was that more than half of the patients we chose to follow needed a second biopsy. Andrykowski and al. proved that 8 months after a benign breast biopsy, women who had undergone the procedure still showed an amount of distress higher than women who did not (Andrykowski et al., 2002). In addition to that, $18 \%$ of the patients followed developed BC. Our results lead us to conclude that $\mathrm{ADH}$ diagnosed by $\mathrm{CNB}$ is a premalignant lesion and is an important risk factor for malignancy in both the short and long term. We therefore recommend surgical excision for all CNBs showing atypical ductal hyperplasia. We propose an algorithm of treatment in figure 3 for all four pathologies. 


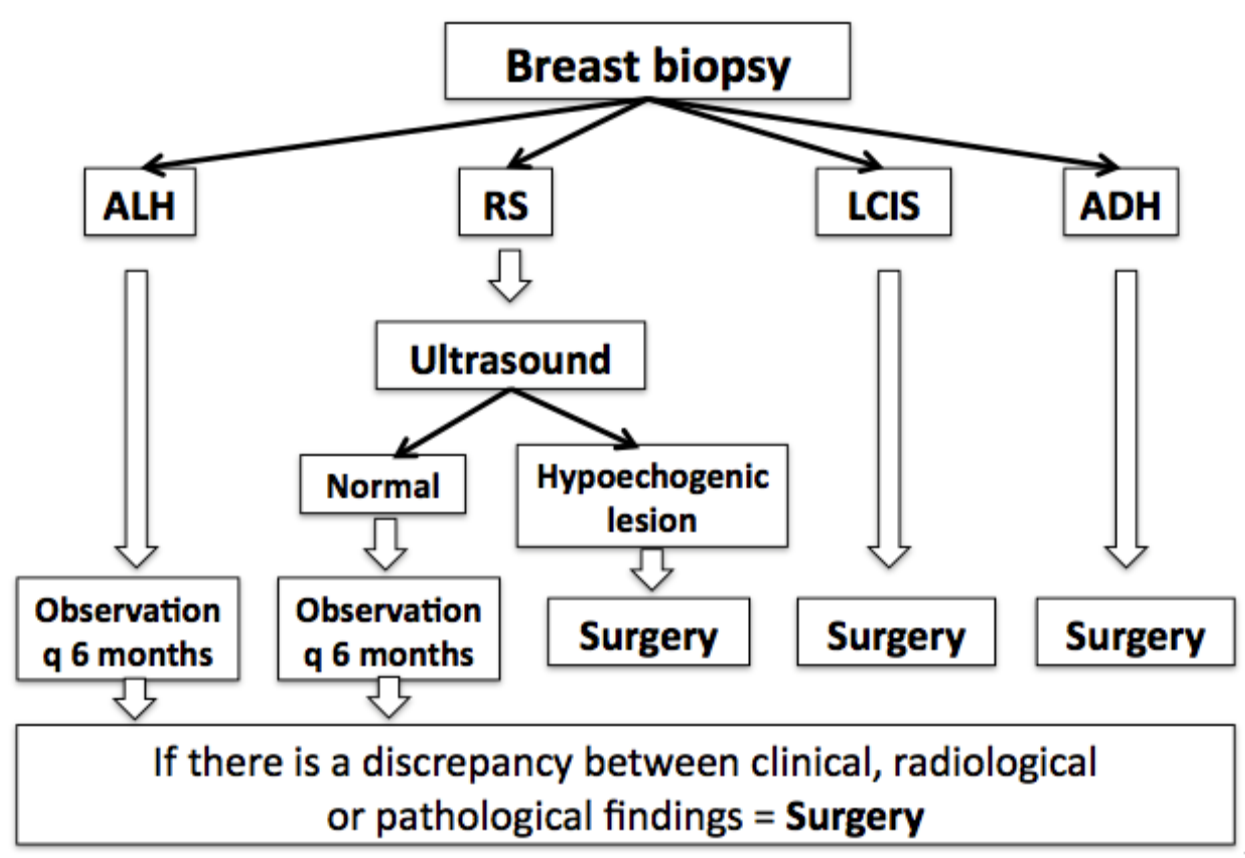

Figure 3. Algorithm of management for ALH, RS, LCIS and ADH

If anytime during investigation there is a discrepancy between clinical, radiological or pathological findings, it should mandate surgical excision. LCIS =lobular carcinoma in situ, ALH= atypical lobular hyperplasia; $\mathrm{RS}=$ radial scar; $\mathrm{ADH}=$ atypical ductal hyperplasia

One limitation of our study is that a low percentage of patients were operated for surgical excision of ADH in our center even though it was commonly accepted. There are two reasons for that. Our institution is a center of referral in radiology for nearby smaller hospitals and some of our patients were operated in those institutions, hence lost to follow up. Another reason is that we took in consideration comorbidities of the patient and had a tendency toward observation if the patient had a short life expectancy. Another limitation is the small number of patients with each pathology, which does not permit us to observe a statistical difference between our groups. This limitation is seen in almost all studies reporting on these conditions, because they are not very common.

\section{Conclusion}

Uncertain malignant lesions are a management challenge for surgeons. After reviewing our upgrade rates and the literature, we suggest that ADH and LCIS at core needle biopsy undergo surgical excision. They present an important risk of initial upgrade. Patients with pathological radial scars should have a breast ultrasound and if the lesion is hypoechogenic, it should be resected. If not, they can be observed. As for ALH, if no clinical or echographic findings are present and the patient has no associated risk factors, we suggest close follow up (See figure 3). 


\section{References}

Andrykowski, M. A., Carpenter, J. S., Studts, J. L., Cordova, M. J., Cunningham, L. L., Beacham, A., et al. (2002). Psychological impact of benign breast biopsy: a longitudinal, comparative study. Health Psychol, 21(5), 485-494.

Berg, J. C., Visscher, D. W., Vierkant, R. A., Pankratz, V. S., Maloney, S. D., Lewis, J. T., et al. (2008). Breast cancer risk in women with radial scars in benign breast biopsies. Breast Cancer Res Treat, 108(2), 167-174. http://dx.doi.org/10.1007/s10549-007-9605-9

Bianchi, S., Giannotti, E., Vanzi, E., Marziali, M., Abdulcadir, D., Boeri, C., et al. (2012). Radial scar without associated atypical epithelial proliferation on image-guided 14-gauge needle core biopsy: Analysis of 49 cases from a single-centre and review of the literature. Breast, 21(2), 159-164. http://dx.doi.org/10.1016/j.breast.2011.09.005

Brogi, E., Murray, M. P., \& Corben, A. D. (2010). Lobular carcinoma, not only a classic. Breast J, 16 Suppl 1, S10-14. http://dx.doi.org/10.1111/j.1524-4741.2010.00994.x

Cangiarella, J., Guth, A., Axelrod, D., Darvishian, F., Singh, B., Simsir, A., et al. (2008). Is surgical excision necessary for the management of atypical lobular hyperplasia and lobular carcinoma in situ diagnosed on core needle biopsy: a report of 38 cases and review of the literature. Arch Pathol Lab Med, 132(6), 979-983. http://dx.doi.org/2007-0446-OAR

Chuba, P. J., Hamre, M. R., Yap, J., Severson, R. K., Lucas, D., Shamsa, F., et al. (2005). Bilateral risk for subsequent breast cancer after lobular carcinoma-in-situ: analysis of surveillance, epidemiology, and end results data. J Clin Oncol, 23(24), 5534-5541. http://dx.doi.org/10.1200/JCO.2005.04.038

Egyed, Z., Pentek, Z., Jaray, B., Kulka, J., Svastics, E., Kas, J., et al. (2008). Radial scar-significant diagnostic challenge. Pathol Oncol Res, 14(2), 123-129. http://dx.doi.org/10.1007/s12253-008-9025-0

Elsheikh, T. M., \& Silverman, J. F. (2005). Follow-up surgical excision is indicated when breast core needle biopsies show atypical lobular hyperplasia or lobular carcinoma in situ: a correlative study of 33 patients with review of the literature. Am J Surg Pathol, 29(4), 534-543. http://dx.doi.org/00000478-200504000-000 17

Flegg, K. M., Flaherty, J. J., Bicknell, A. M., \& Jain, S. (2010). Surgical outcomes of borderline breast lesions detected by needle biopsy in a breast screening program. World $J$ Surg Oncol, 8, 78. http://dx.doi.org/10.1186/1477-7819-8-78

Haagensen, C. D., Lane, N., Lattes, R., \& Bodian, C. (1978). Lobular neoplasia (so-called lobular carcinoma in situ) of the breast. Cancer, 42(2), 737-769.

Hunt, R. J., Steel, J. R., Porter, G. J., Holgate, C. S., \& Watkins, R. M. (2012). Lesions of uncertain malignant potential (B3) on core biopsy in the NHS Breast Screening Programme: is the screening round relevant? Ann R Coll Surg Engl, 94(2), 108-111. http://dx.doi.org/10.1308/003588412X13171221498460

Jackman, R. J., Birdwell, R. L., \& Ikeda, D. M. (2002). Atypical ductal hyperplasia: can some lesions be defined as probably benign after stereotactic 11-gauge vacuum-assisted biopsy, eliminating the recommendation for surgical excision? Radiology, 224(2), 548-554.

Jacobs, T. W., Byrne, C., Colditz, G., Connolly, J. L., \& Schnitt, S. J. (1999). Radial scars in benign breast-biopsy specimens and the risk of breast cancer. $N$ Engl J Med, 340(6), 430-436.

Lavoue, V., Graesslin, O., Classe, J. M., Fondrinier, E., Angibeau, H., \& Leveque, J. (2007). Management of lobular neoplasia diagnosed by core needle biopsy: study of 52 biopsies with follow-up surgical excision. Breast, 16(5), 533-539. http://dx.doi.org/10.1016/j.breast.2007.04.005

Liberman, L., Holland, A. E., Marjan, D., Murray, M. P., Bartella, L., Morris, E. A., et al. (2007). Underestimation of atypical ductal hyperplasia at MRI-guided 9-gauge vacuum-assisted breast biopsy. AJR Am J Roentgenol, 188(3), 684-690. http://dx.doi.org/10.2214/AJR.06.0809

Liberman, L., Sama, M., Susnik, B., Rosen, P. P., LaTrenta, L. R., Morris, E. A., et al. (1999). Lobular carcinoma in situ at percutaneous breast biopsy: surgical biopsy findings. AJR Am J Roentgenol, 173(2), 291-299.

Londero, V., Zuiani, C., Linda, A., Vianello, E., Furlan, A., \& Bazzocchi, M. (2008). Lobular neoplasia: core needle breast biopsy underestimation of malignancy in relation to radiologic and pathologic features. Breast, 17(6), 623-630. http://dx.doi.org/10.1016/j.breast.2008.05.007 
Middleton, L. P., Perkins, G. H., Tucker, S. L., Sahin, A. A., \& Singletary, S. E. (2007). Expression of ERalpha and ERbeta in lobular carcinoma in situ. Histopathology, 50(7), 875-880. http://dx.doi.org/10.1111/j.1365-2559.2007.02689.x

Page, D. L., Kidd, T. E., Jr., Dupont, W. D., Simpson, J. F., \& Rogers, L. W. (1991). Lobular neoplasia of the breast: higher risk for subsequent invasive cancer predicted by more extensive disease. Hum Pathol, 22(12), 1232-1239.

Pagni, F., Bosisio, F. M., Salvioni, D., Colombo, P., Leone, B. E., \& Di Bella, C. (2012). Application of the British National Health Service Breast Cancer Screening Programme classification in 226 breast core needle biopsies: correlation with resected specimens. Ann Diagn Pathol, 16(2), 112-118. http://dx.doi.org/10.1016/j.anndiagpath.2011.08.003

Purcell, C. A., \& Norris, H. J. (1998). Intraductal proliferations of the breast: a review of histologic criteria for atypical intraductal hyperplasia and ductal carcinoma in situ, including apocrine and papillary lesions. Ann Diagn Pathol, 2(2), 135-145. http://dx.doi.org/S1092-9134(98)80051-8

Rosen, P. (2001). Rosen breast's pathology, 2nd edition (Vol. xviii). Philadelphia: lippincott williams and wilkins publisher.

Sanders, M. E., Page, D. L., Simpson, J. F., Schuyler, P. A., Dale Plummer, W., \& Dupont, W. D. (2006). Interdependence of radial scar and proliferative disease with respect to invasive breast carcinoma risk in patients with benign breast biopsies. Cancer, 106(7), 1453-1461. http://dx.doi.org/10.1002/cncr. 21730

Schnitt, S. J. C. L. (2009). Biopsy Interpretation of the Breast

Tavassoli, F. A. E. V. (2009). In Tumors of the Mammary Gland, AFIP Atlas of Tumor Pathology.

Vos, C. B., Cleton-Jansen, A. M., Berx, G., de Leeuw, W. J., ter Haar, N. T., van Roy, F., et al. (1997). E-cadherin inactivation in lobular carcinoma in situ of the breast: an early event in tumorigenesis. Br J Cancer, 76(9), 1131-1133. 